\title{
A Benchmark Approach to Portfolio Optimization under Partial Information
}

\author{
Eckhard Platen ${ }^{1}$ and Wolfgang J. Runggaldier ${ }^{2}$
}

January 15, 2007

\begin{abstract}
This paper proposes a filtering methodology for portfolio optimization when some factors of the underlying model are only partially observed. The level of information is given by the observed quantities that are here supposed to be the primary securities and empirical log-price covariations. For a given level of information we determine the growth optimal portfolio, identify locally optimal portfolios that are located on a corresponding Markowitz efficient frontier and present an approach for expected utility maximization. We also present an expected utility indifference pricing approach under partial information for the pricing of nonreplicable contracts. This results in a real world pricing formula under partial information that turns out to be independent of the subjective utility of the investor and for which an equivalent risk neutral probability measure need not exist.
\end{abstract}

1991 Mathematics Subject Classification: primary 90A12; secondary 60G30, 62P20. JEL Classification: G10, G13

Key words and phrases: portfolio optimization, partial information, filtering, growth optimal portfolio, expected utility maximization, utility indifference pricing, real world pricing formula.

\footnotetext{
${ }^{1}$ University of Technology Sydney, School of Finance \& Economics and Department of Mathematical Sciences, PO Box 123, Broadway, NSW, 2007, Australia

${ }^{2}$ Department of Pure and Applied Mathematics, University of Padova Via Trieste 63, 35121 Padova, ITALY
} 


\section{Introduction}

In finance one faces often the situation that not all quantities that appear in market models are fully observable. Examples for such models with partial observation can be found, for instance, in Lakner (1995), Elliott, Lahaie \& Madan (1997), Elliott, Fischer \& Platen (1999), Babbs \& Nowman (1999), Frey \& Runggaldier (1999, 2001), Karatzas \& Zhao (2001), Pham \& Quenez (2001), Nagai \& Peng (2002), Bhar, Chiarella \& Runggaldier (2004), Haussmann \& Sass (2004), Kirch \& Runggaldier (2004), Landen (2000), Runggaldier (2004), Gombani, Jaschke \& Runggaldier (2005), Cvitanić, Rozovskii \& Zaliapin (2006) and Bäuerle \& Rieder (2007). For instance, of particular interest are the market prices of risk, which are important hidden quantities that the investors need for portfolio optimization.

In Platen \& Runggaldier (2005) a benchmark approach to filtering in finance for the pricing and hedging in incomplete markets has been proposed using the framework presented in Platen \& Heath (2006). It turns out that these results are useful also in the context of portfolio optimization under partial information. The current paper describes a general methodology for applying filtering methods to portfolio optimization when some of the factors are hidden. The level of information is given by the number of observed quantities that are here supposed to be the primary securities and empirical log-price covariations. For a given level of information we determine the growth optimal portfolio using filtered values of the hidden factors. As it should be, the expected optimal growth rate under full information turns out to be larger than that under partial information. The family of locally optimal portfolios is identified under partial information, whereby one invests only in the growth optimal portfolio and in the savings account. Its members have all the same Sharpe ratio and are located on a corresponding Markowitz efficient frontier. Furthermore, we present a general approach to expected utility maximization under partial information that results in an optimal strategy, yielding locally optimal portfolios.

Finally we present an expected utility indifference pricing approach for the pricing of nonreplicable contracts under partial information that is consistent with both portfolio optimization and derivative pricing under complete information. It results in a real world pricing formula under partial information that turns out to be independent of the subjective utility of the investor and for which an equivalent risk neutral probability measure need not exist.

The paper is organized as follows: Section 2 describes the market model in form of a system of stochastic differential equations for the factors. Section 3 clarifies the roles of the observable and hidden factors. In Section 4 the GOP will be constructed for a given level of information. Locally optimal portfolios will be studied in Section 5. The maximization of expected utility under partial observation will be performed in Section 6. Finally, in Section 7 we consider utility indifference pricing under partial information. 


\section{The Market Model}

We consider a market with $d+1$ primary securities that we shall describe with a factor model as follows:

Let there be given a filtered probability space $(\Omega, \mathcal{A}, \underline{\mathcal{A}}, P)$ with $\underline{\mathcal{A}}=\left(\mathcal{A}_{t}\right)_{t \in \Re_{+}}$ denoting its filtration that satisfies the usual conditions. Consider $d$ independent $(\underline{\mathcal{A}}, P)$-Wiener processes $v^{i}=\left\{v_{t}^{i}, t \in \Re_{+}\right\}, i \in\{1,2, \ldots, d\}$, that model the traded uncertainty. Let $\boldsymbol{X}_{t}=\left(X_{t}^{1}, X_{t}^{2}, \ldots, X_{t}^{m}\right)^{\top} \in \Re^{m}$ be the vector of hidden factors. We assume the hidden factors to be neither fully known nor fully observable. Furthermore, let $\theta_{t}^{j}$ be the $j$-th, $j \in\{1,2, \ldots, d\}$, market price of risk that is supposed to be of the form $\theta_{t}^{j}=\theta^{j}\left(t, \boldsymbol{X}_{t}\right)$. We assume that the total market price of risk $\left|\boldsymbol{\theta}_{t}\right|=\left(\sum_{j=1}^{d}\left(\theta_{t}^{j}\right)^{2}\right)^{\frac{1}{2}}$ is strictly positive and finite, almost surely for all $t \in \Re_{+}$.

Let $\boldsymbol{Y}=\left\{\boldsymbol{Y}_{t}, t \in \Re\right\}$ be a $k$-dimensional vector process, $k \geq d$, of observable factors, which we will specify later. The vector of global factors is then obtained as

$$
\boldsymbol{Z}_{t}=\left(Z_{t}^{1}, \ldots, Z_{t}^{m+k}\right)^{\top}=\left(X_{t}^{1}, \ldots, X_{t}^{m}, Y_{t}^{1}, \ldots, Y_{t}^{k}\right)^{\top} .
$$

The value of the savings account at time $t \in \Re$ is denoted by $S_{t}^{0}$, where

$$
S_{t}^{0}=\exp \left\{\int_{0}^{t} r_{s} d s\right\}
$$

with $r=\left\{r_{t}, t \in \Re_{+}\right\}$denoting the observed adapted short rate process.

The discounted, with respect to $S_{t}^{0}$, values of the $d$ risky primary security accounts are assumed to satisfy the SDE

$$
d \bar{S}_{t}^{j}=d\left(\frac{S_{t}^{j}}{S_{t}^{0}}\right)=\bar{S}_{t}^{j}\left(\sum_{i=1}^{d} b^{j, i}\left(t, \boldsymbol{Y}_{t}\right)\left(\theta^{i}\left(t, \boldsymbol{X}_{t}\right) d t+d v_{t}^{i}\right)\right)
$$

for $j \in\{1,2, \ldots, d\}$ and $t \in \Re_{+}$. These primary security accounts are typically stocks with all dividends reinvested. Without further mentioning we make the following standing assumption:

Assumption 1 We assume that the volatility matrix $\boldsymbol{b}_{t}=\boldsymbol{b}\left(t, \boldsymbol{Y}_{t}\right)=$ $\left[b^{j, i}\left(t, \boldsymbol{Y}_{t}\right)\right]_{j, i=1}^{d}$ is invertible for all $t \in \Re_{+}$.

There exists then a growth optimal portfolio (GOP) $S^{\delta_{*}}$, obtained from investing in the $d+1$ primary security accounts according to a self-financing strategy $\delta_{*}$. It is known, see Platen (2005), Platen \& Heath (2006) and (30) in Section 4 below, that its discounted value $\bar{S}_{t}^{\delta_{*}}$ at time $t$ satisfies under full information the SDE

$$
d \bar{S}_{t}^{\delta_{*}}=\bar{S}_{t}^{\delta_{*}}\left(\sum_{i=1}^{d} \theta^{i}\left(t, \boldsymbol{X}_{t}\right)\left(\theta^{i}\left(t, \boldsymbol{X}_{t}\right) d t+d v_{t}^{i}\right)\right)
$$


for $t \in \Re_{+}$. The dynamics of the GOP depends on the market prices of risk and, thus, also on the hidden factor process $\boldsymbol{X}=\left\{\boldsymbol{X}_{t}=\left(X_{t}^{1}, \ldots, X_{t}^{m}\right)^{\top}, t \in\right.$ $\left.\Re_{+}\right\}$. While the assets that make up the GOP will be given by the primary security accounts, the value of the GOP depends also on the fractions invested in these securities. These fractions depend in turn on the actually available level of information with respect to the hidden factor process $\boldsymbol{X}$. According to the available level of information we shall determine in Section 3 the fractions invested in the individual primary securities for the GOP. Once these fractions are obtained, the GOP can be constructed using these weights.

Concerning the available level of information we consider, for illustration, the following situation, where other cases can be treated similarly :

In addition to the observed primary security account prices there are other observables that provide information about hidden factors. Here we shall, in particular, consider empirical log-price covariations between primary security accounts and the GOP. These are essential for portfolio optimization since they contain information about the hidden market prices of risk. We make the assumption that the observed empirical log-price covariations represent approximations of the actual, but hidden, theoretical covariations. The approximative, perturbed nature of empirical log-price covariations derives from the fact that these are determined over an equidistant discrete time grid with step size $\Delta$ by summing products of log-price increments. Other observed quantities that may depend on hidden processes can be treated similarly.

Let now

$$
z_{t}^{j}=\left[\ln \left(\bar{S}^{j}\right), \ln \left(\bar{S}^{\delta_{*}}\right)\right]_{\Delta, t}=\sum_{\ell=1}^{\left[\frac{t}{\Delta}\right]}\left(\ln \left(\bar{S}_{t_{\ell}}^{j}\right)-\ln \left(\bar{S}_{t_{\ell-1}}^{j}\right)\right)\left(\ln \left(\bar{S}_{t_{\ell}}^{\delta_{*}}\right)-\ln \left(\bar{S}_{t_{\ell-1}}^{\delta_{*}}\right)\right)
$$

for $j \in\{1,2, \ldots, d\}$ denote the empirical covariation of the logarithm of the $j$ th primary security account with the logarithm of the GOP up to time $t \in \Re$, with $t_{\ell}=\ell \Delta, \ell \in\{1,2, \ldots\}$. Here $[a]$ denotes the largest integer not greater than or equal to $a$.

Define

$$
B^{j}\left(t, \boldsymbol{Z}_{t}\right)=\sum_{i=1}^{d} b^{j, i}\left(t, \boldsymbol{Y}_{t}\right) \theta^{i}\left(t, \boldsymbol{X}_{t}\right)
$$

then, given the SDEs (3) and (4) for the discounted primary security accounts and the GOP, the hidden theoretical values corresponding to the empirical covariations $z_{t}^{j}$ can be expressed as

$$
\left[\ln \left(\bar{S}^{j}\right), \ln \left(\bar{S}^{\delta_{*}}\right)\right]_{t}=\int_{0}^{t} B^{j}\left(s, \boldsymbol{Z}_{s}\right) d s
$$

for $j \in\{1,2, \ldots, d\}, t \in \Re$. 
The evolution of the observed empirical covariations are now modeled with some simple perturbation as follows:

$$
d z_{t}^{j}=B^{j}\left(t, Z_{t}\right) d t+d \eta_{t}^{j}
$$

for $j \in\{1,2, \ldots, d\}$. Here $\eta^{j}=\left\{\eta_{t}^{j}, t \in \Re_{+}\right\}, j \in\{1,2, \ldots, d\}$, are independent standard $(\underline{\mathcal{A}}, P)$-Wiener processes on $(\Omega, \mathcal{A}, \underline{\mathcal{A}}, P)$, independent among themselves and from the Wiener processes $v^{1}, v^{2}, \ldots, v^{d}$. Note that in the SDE (8) we introduce some noise into the empirical covariations but can also create some feedback into the market prices of risk $\theta^{i}\left(t, \boldsymbol{X}_{t}\right)$ in (3), as will become clear later. Of course, alternative ways for modeling the observed empirical covariations in relation to their hidden counterparts can be pursued. The aim of this paper, however, is to describe in a simple manner for a basic example the main steps and results that allow such kind of modeling in the context of portfolio optimization. Note that this is highly relevant for portfolio selection since it demonstrates how to gain access to estimates for the market prices of risk.

\section{Observable and Hidden Factors}

The process $\boldsymbol{X}=\left\{\boldsymbol{X}_{t}, t \in \Re_{+}\right\}$is assumed to be the unobservable vector process component of the factors, while the discounted primary security account values and the empirical covariations are observable.

Let then

$$
\boldsymbol{Y}_{t}=\left(\bar{S}_{t}^{1}, \ldots, \bar{S}_{t}^{d}, z_{t}^{1}, \ldots, z_{t}^{d}\right)^{\top}
$$

denote the vector of observable factors at time $t$. Introduce the $d$-vectors

$$
\boldsymbol{\theta}\left(t, \boldsymbol{X}_{t}\right)=\left(\theta^{1}\left(t, \boldsymbol{X}_{t}\right), \ldots, \theta^{d}\left(t, \boldsymbol{X}_{t}\right)\right)^{\top}
$$

and

$$
\boldsymbol{B}\left(t, \boldsymbol{Z}_{t}\right)=\left(B^{1}\left(t, \boldsymbol{Z}_{t}\right), \ldots, B^{d}\left(t, \boldsymbol{Z}_{t}\right)\right)^{\top}
$$

Finally, we define the $k=2 d$-dimensional $(\underline{\mathcal{A}}, P)$-Wiener process

$$
\boldsymbol{W}=\left\{\boldsymbol{W}_{t}=\left(v_{t}^{1}, \ldots, v_{t}^{d}, \eta_{t}^{1}, \ldots, \eta_{t}^{d}\right)^{\top}, t \in \Re_{+}\right\} .
$$

On the basis of (9), (3) and (8) we can write the dynamics of $\boldsymbol{Y}_{t}$ as

$$
\begin{aligned}
d \boldsymbol{Y}_{t} & =\left(\begin{array}{c}
\operatorname{diag}\left(\overline{\boldsymbol{S}}_{t}\right) \boldsymbol{b}\left(t, \boldsymbol{Y}_{t}\right) \boldsymbol{\theta}\left(t, \boldsymbol{X}_{t}\right) \\
\boldsymbol{B}\left(t, \boldsymbol{Z}_{t}\right)
\end{array}\right) d t+\left(\begin{array}{cc}
\operatorname{diag}\left(\overline{\boldsymbol{S}}_{t}\right) \boldsymbol{b}\left(t, \boldsymbol{Y}_{t}\right) & 0 \\
0 & \boldsymbol{I}
\end{array}\right) d \boldsymbol{W}_{t} \\
& =\boldsymbol{F}\left(t, \boldsymbol{Z}_{t}\right) d t+\boldsymbol{G}\left(t, \boldsymbol{Y}_{t}\right) d \boldsymbol{W}_{t},
\end{aligned}
$$

defining implicitly the functions $\boldsymbol{F}(\cdot)$ and $\boldsymbol{G}(\cdot)$, where $\boldsymbol{I}$ is the unit matrix. The vector of global factors equals by $(1) \boldsymbol{Z}_{t}=\left(X_{t}^{1}, \ldots, X_{t}^{m}, Y_{t}^{1}, \ldots, Y_{t}^{k}\right)^{\top}$ having 
dimension $m+k$, of which the first $m$ components are not observable while the remaining $k$ are fully observable. The level of information can be characterized by the integer $k$ that amounts in our specific case to $k=2 d$, parameterized by the number $d$ of risky primary security accounts.

Whatever the situation, for given $k$ we shall denote by $\underline{\mathcal{A}}^{k}=\left(\mathcal{A}_{t}^{k}\right)_{t \in \Re_{+}}$with

$$
\mathcal{A}_{t}^{k}=\sigma\left\{\boldsymbol{Y}_{s}, s \leq t\right\} \subseteq \mathcal{A}_{t}
$$

for $t \in \Re_{+}$, the subfiltration corresponding to the available information level.

To better model the market at a given level of information $k$ we shall, by analogy to Platen \& Runggaldier (2005), assume that the conditional filter distribution

$$
p\left(\boldsymbol{X}_{t} \mid \mathcal{A}_{t}^{k}\right)=p\left(\boldsymbol{X}_{t} \mid \sigma\left\{\boldsymbol{Y}_{s}, s \leq t\right\}\right)
$$

is parameterized by a fixed, finite number of parameter processes

$$
\boldsymbol{\xi}_{t}=\left(\xi_{t}^{1}, \ldots, \xi_{t}^{q}\right)^{\top}
$$

obtained from a finite dimensional filter. This allows us to consider a new $(k+q)$ dimensional vector of global factors

$$
\tilde{\boldsymbol{Z}}_{t}=\left(Y_{t}^{1}, \ldots, Y_{t}^{k}, \xi_{t}^{1}, \ldots, \xi_{t}^{q}\right)^{\top}
$$

of which all components are observable or computable from observables. Furthermore, we shall assume that the filter state $\boldsymbol{\xi}_{t}=\left(\xi_{t}^{1}, \ldots, \xi_{t}^{q}\right)^{\top}$ at time $t$ satisfies the vector SDE

$$
d \boldsymbol{\xi}_{t}=\boldsymbol{H}\left(t, \tilde{\boldsymbol{Z}}_{t}\right) d t+\boldsymbol{K}\left(t, \tilde{\boldsymbol{Z}}_{t}\right) d \boldsymbol{Y}_{t} .
$$

We refer to Platen \& Runggaldier (2005) for examples and a discussion of this assumption.

The possibility of finding a finite dimensional filter, evidently requires the existence of a regular solution of the corresponding filtering problem, i.e. that of determining the conditional distribution $p\left(\boldsymbol{X}_{t} \mid \mathcal{A}_{t}^{k}\right)$.

We can readily adapt the results of Proposition 2.3 in Platen \& Runggaldier $(2005)$ to our case. Consequently, there exists a $k$-dimensional, $\underline{\mathcal{A}}^{k}$-adapted $\left(\underline{\mathcal{A}}^{k}, P\right)$-Wiener process $\tilde{\boldsymbol{W}}=\left\{\tilde{\boldsymbol{W}}_{t}, t \in \Re_{+}\right\}$such that the process $\boldsymbol{Y}$ of observable factors satisfies the SDE

$$
d \boldsymbol{Y}_{t}=\tilde{\boldsymbol{F}}\left(t, \tilde{\boldsymbol{Z}}_{t}\right) d t+\tilde{\boldsymbol{G}}\left(t, \tilde{\boldsymbol{Z}}_{t}\right) d \tilde{\boldsymbol{W}}_{t}
$$

where

$$
\tilde{\boldsymbol{F}}\left(t, \tilde{\boldsymbol{Z}}_{t}\right)=E\left(\boldsymbol{F}\left(t, \boldsymbol{Z}_{t}\right) \mid \mathcal{A}_{t}^{k}\right)
$$

and

$$
\tilde{\boldsymbol{G}}\left(t, \tilde{\boldsymbol{Z}}_{t}\right)=E\left(\boldsymbol{G}\left(t, \boldsymbol{Y}_{t}\right) \mid \mathcal{A}_{t}^{k}\right)=G\left(t, \boldsymbol{Y}_{t}\right)
$$


We refer to (13) for the definition of $\boldsymbol{F}(\cdot)$ and $\boldsymbol{G}(\cdot)$. Replacing the expression of $d \boldsymbol{Y}_{t}$ from (18) in (17) we obtain

$$
d \boldsymbol{\xi}_{t}=\tilde{\boldsymbol{H}}\left(t, \tilde{\boldsymbol{Z}}_{t}\right) d t+\tilde{\boldsymbol{K}}\left(t, \tilde{\boldsymbol{Z}}_{t}\right) d \tilde{\boldsymbol{W}}_{t}
$$

with

$$
\tilde{\boldsymbol{H}}\left(t, \tilde{\boldsymbol{Z}}_{t}\right)=\boldsymbol{H}\left(t, \tilde{\boldsymbol{Z}}_{t}\right)+\boldsymbol{K}\left(t, \tilde{\boldsymbol{Z}}_{t}\right) \tilde{\boldsymbol{F}}\left(t, \tilde{\boldsymbol{Z}}_{t}\right)
$$

and

$$
\tilde{\boldsymbol{K}}\left(t, \tilde{\boldsymbol{Z}}_{t}\right)=\boldsymbol{K}\left(t, \tilde{\boldsymbol{Z}}_{t}\right) \boldsymbol{G}\left(t, \boldsymbol{Y}_{t}\right)
$$

Combining (18) and (21) we see that the observable factor vector $\tilde{\boldsymbol{Z}}_{t}$ given in (16) forms a Markov process satisfying an SDE of the form

$$
d \tilde{Z}_{t}^{j}=\alpha^{j}\left(t, \tilde{\boldsymbol{Z}}_{t}\right) d t+\sum_{i=1}^{k} \beta^{j, i}\left(t, \tilde{\boldsymbol{Z}}_{t}\right) d \tilde{W}_{t}^{i}
$$

for $j \in\{1,2, \ldots, k+q\}$.

We remark that the first $d$ components $\tilde{Z}_{t}^{j}, j \in\{1,2, \ldots, d\}$, of $\tilde{Z}_{t}$ coincide with the discounted primary security account prices $\bar{S}_{t}^{j}$ and, given the particular blockdiagonal structure of the matrix $\boldsymbol{G}\left(t, \boldsymbol{Y}_{t}\right)$ in (13), these first $d$ primary security accounts are driven only by the first $d$ components of $\tilde{\boldsymbol{W}}$, the traded noise, so that

$$
\beta^{j, i}\left(t, \tilde{Z}_{t}\right)=0
$$

for $j \leq d$ and $i>d$. Actually, it follows from (13) and the adaptation of the proof of Proposition 2.3 in Platen \& Runggaldier (2005) to our case that for $Y_{t}^{j}=\bar{S}_{t}^{j}$ we have

$$
d \bar{S}_{t}^{j}=\bar{S}_{t}^{j}\left(\tilde{B}^{j}\left(t, \tilde{\boldsymbol{Z}}_{t}\right) d t+\sum_{i=1}^{d} \tilde{b}^{j, i}\left(t, \tilde{\boldsymbol{Z}}_{t}\right) d \tilde{W}_{t}^{i}\right)
$$

where, for $j \leq d$,

$$
\tilde{B}^{j}\left(t, \tilde{\boldsymbol{Z}}_{t}\right)=E\left(B^{j}\left(t, \boldsymbol{Z}_{t}\right) \mid \mathcal{A}_{t}^{k}\right)
$$

with $B^{j}\left(t, \boldsymbol{Z}_{t}\right)$ as in (6) and

$$
\tilde{b}^{j, i}\left(t, \tilde{\boldsymbol{Z}}_{t}\right)=E\left(b^{j, i}\left(t, \boldsymbol{Y}_{t}\right) \mid \mathcal{A}_{t}^{k}\right)=b^{j, i}\left(t, \boldsymbol{Y}_{t}\right)
$$

with the volatility $b^{j, i}\left(t, \boldsymbol{Y}_{t}\right)$ appearing in (3). It is important to note that in (23) the risk premia are $\underline{\mathcal{A}}^{k}$-adapted while in (3) this is not the case.

\section{Maximizing the Growth Rate}

We first recall the situation under full information. Since under full information the discounted values of the primary security account prices $\bar{S}_{t}^{j}$ satisfy (3), the discounted value of a self-financing portfolio corresponding to an investment 
strategy $\boldsymbol{\delta}=\left\{\boldsymbol{\delta}_{t}=\left(\delta_{t}^{0}, \delta_{t}^{1}, \ldots, \delta_{t}^{d}\right)^{\top}, t \in \Re_{+}\right\}$expressing the respective numbers of units invested in the various assets, satisfies the SDE

$$
d \bar{S}_{t}^{\delta}=\sum_{j=1}^{d} \delta_{t}^{j} \bar{S}_{t}^{j} \sum_{i=1}^{d} b^{j, i}\left(t, \boldsymbol{Y}_{t}\right)\left(\theta^{i}\left(t, \boldsymbol{X}_{t}\right) d t+d v_{t}^{i}\right)
$$

or, when using the fraction $\pi_{\delta, t}^{j}=\frac{\delta_{t}^{j} \bar{S}_{t}^{j}}{\bar{S}_{t}^{\delta}}$ the equivalent $\mathrm{SDE}$

$$
d \bar{S}_{t}^{\delta}=\bar{S}_{t}^{\delta} \sum_{j=1}^{d} \pi_{\delta, t}^{j} \sum_{i=1}^{d} b^{j, i}\left(t, \boldsymbol{Y}_{t}\right)\left(\theta^{i}\left(t, \boldsymbol{X}_{t}\right) d t+d v_{t}^{i}\right)
$$

From here it follows, see, e.g. Platen \& Heath (2006), that the corresponding growth rate under full information equals

$$
\bar{g}_{t}^{\delta}=\sum_{i=1}^{d}\left(\sum_{j=1}^{d} \pi_{\delta, t}^{j} b^{j, i}\left(t, \boldsymbol{Y}_{t}\right) \theta^{i}\left(t, \boldsymbol{X}_{t}\right)-\frac{1}{2}\left(\sum_{\ell=1}^{d} \pi_{\delta, t}^{\ell} b^{\ell, i}\left(t, \boldsymbol{Y}_{t}\right)\right)^{2}\right)
$$

We have now the following result.

Proposition 2 The investment strategy of a GOP is under full information given by the fractions

$$
\pi_{\delta_{*}, t}^{j}=\sum_{i=1}^{d} \theta^{i}\left(t, \boldsymbol{X}_{t}\right)\left(b^{-1}\right)^{j, i}\left(t, \boldsymbol{Y}_{t}\right)
$$

where by $\left(b^{-1}\right)^{j, i}\left(t, \boldsymbol{Y}_{t}\right)$ we denote the $(j, i)$ th component of the inverse of the volatility matrix $\boldsymbol{b}_{t}=\boldsymbol{b}\left(t, \boldsymbol{Y}_{t}\right)$. Furthermore, the discounted value of the GOP satisfies

$$
d \bar{S}_{t}^{\delta_{*}}=\bar{S}_{t}^{\delta_{*}}\left(\sum_{i=1}^{d} \theta^{i}\left(t, \boldsymbol{X}_{t}\right)\left(\theta^{i}\left(t, \boldsymbol{X}_{t}\right) d t+d v_{t}^{i}\right)\right) .
$$

Finally, the benchmarked portfolio value $\frac{S_{t}^{\delta}}{S_{t}^{\delta_{*}}}$ corresponding to a self-financing strategy $\boldsymbol{\delta}$ is an $(\underline{\mathcal{A}}, P)$-local martingale satisfying

$$
\begin{aligned}
d\left(\frac{S_{t}^{\delta}}{S_{t}^{\delta_{*}}}\right) & =\frac{S_{t}^{\delta}}{S_{t}^{\delta_{*}}} \sum_{j=1}^{d} \delta_{t}^{j} \sum_{i=1}^{d}\left(b^{j, i}\left(t, \boldsymbol{Y}_{t}\right)-\theta^{i}\left(t, \boldsymbol{X}_{t}\right)\right) d v_{t}^{i} \\
& =\frac{S_{t}^{\delta}}{S_{t}^{\delta_{*}}} \sum_{j=1}^{d} \pi_{\delta, t}^{j} \sum_{i=1}^{d}\left(b^{j, i}\left(t, \boldsymbol{Y}_{t}\right)-\theta^{i}\left(t, \boldsymbol{X}_{t}\right)\right) d v_{t}^{i}
\end{aligned}
$$


Proof: Relation (29) is an immediate consequence of the first order conditions applied to (28). Relation (30) then results by combining (27) and (29). Finally, relation (31) is obtained from (27) and (30) by Itô's formula.

We are now interested in obtaining corresponding results for the case under partial information characterized by the filtration $\mathcal{A}^{k}$. Recall that, to be practicable, the strategies have to be adapted to $\underline{\mathcal{A}}^{k}$ since, in practice, they can depend only on available information.

Notice first that for $j \leq d$ it holds $\bar{S}_{t}^{j}=Y_{t}^{j}$ so that, under the information level corresponding to $\mathcal{A}_{t}^{k}$, we have the $\operatorname{SDE}(23)$. For an investment strategy $\delta$ the corresponding discounted portfolio value $\bar{S}_{t}^{\delta}$ satisfies then by (25) the SDE

$$
d \bar{S}_{t}^{\delta}=\bar{S}_{t}^{\delta} \sum_{j=1}^{d} \pi_{\delta, t}^{j, k}\left(\tilde{B}^{j}\left(t, \tilde{\boldsymbol{Z}}_{t}\right) d t+\sum_{i=1}^{d} b^{j, i}\left(t, \boldsymbol{Y}_{t}\right) d \tilde{W}_{t}^{i}\right)
$$

leading to an $\underline{\mathcal{A}}^{k}$-adapted growth rate

$$
\bar{g}_{t}^{\delta, k}=\sum_{j=1}^{d} \pi_{\delta, t}^{j, k} \tilde{B}^{j}\left(t, \tilde{\boldsymbol{Z}}_{t}\right)-\frac{1}{2} \sum_{i=1}^{d}\left(\sum_{\ell=1}^{d} \pi_{\delta, t}^{\ell, k} b^{\ell, i}\left(t, \boldsymbol{Y}_{t}\right)\right)^{2}
$$

To obtain the GOP strategy under $\underline{\mathcal{A}}^{k}$ let

$$
A^{j, i}\left(t, \boldsymbol{Y}_{t}\right)=\sum_{\ell=1}^{d} b^{j, \ell}\left(t, \boldsymbol{Y}_{t}\right) b^{i, \ell}\left(t, \boldsymbol{Y}_{t}\right)
$$

for $j, i \in\{1,2, \ldots, d\}$. Using again the first order conditions, this time on (33), we immediately obtain the following result:

Proposition 3 The investment strategy of the GOP $\bar{S}_{t}^{\delta_{*}, k}$ is, under the partial information corresponding to $\underline{\mathcal{A}}^{k}$ given by the fractions

$$
\pi_{\delta_{*}, t}^{j, k}=\sum_{i=1}^{d} \tilde{B}^{i}\left(t, \tilde{\boldsymbol{Z}}_{t}\right)\left(A^{-1}\right)^{j, i}\left(t, \boldsymbol{Y}_{t}\right)
$$

where $\left(A^{-1}\right)^{j, i}$ is the $(j, i)$ th element of the inverse of the matrix with elements $A^{j, i}$ as in (34) and $\tilde{B}^{i}$ is as in (24).

The situation is, thus, somewhat different from the case of full information. We still have reasonably simple formulas like those in (30) and (31) for the GOP and the benchmarked portfolio values, as will be shown below. Notice that by (32) and (35) the discounted value of the growth optimal portfolio $\bar{S}_{t}^{\delta_{*}, k}$ under $\underline{\mathcal{A}}^{k}$ is completely determined by the evolution of the observed factor process $\tilde{Z}$, which includes the characterization of the filtered values of the hidden factors. Since 
the latter process is Markov, see (22), it follows from Corollary 2.5 in Platen \& Runggaldier (2005) that for a given function $g: \Re^{m+k} \rightarrow \Re$ there exists a suitable function $u:[0, T] \times \Re^{k+q} \rightarrow \Re$ such that

$$
u\left(t, \tilde{\boldsymbol{Z}}_{t}\right)=E\left(g\left(\boldsymbol{Z}_{T}\right) \mid \mathcal{A}_{t}^{k}\right)
$$

for $t \leq T<\infty$.

This yields the following result:

Corollary 4 The SDE (3) for the discounted primary security accounts can be written in the form

$$
d \bar{S}_{t}^{j}=\bar{S}_{t}^{j}\left(\sum_{i=1}^{d} b^{j, i}\left(t, \boldsymbol{Y}_{t}\right)\left(\tilde{\theta}^{i}\left(t, \tilde{\boldsymbol{Z}}_{t}\right) d t+d \tilde{W}_{t}^{i}\right)\right)
$$

with

$$
\tilde{\theta}^{i}\left(t, \tilde{\boldsymbol{Z}}_{t}\right)=E\left(\theta^{i}\left(t, \boldsymbol{X}_{t}\right) \mid \mathcal{A}_{t}^{k}\right)
$$

Consequently, in correspondence to (27)-(31) we obtain in this case for a discounted portfolio $\bar{S}^{\delta}$ the $S D E$

$$
d \bar{S}_{t}^{\delta}=\bar{S}_{t}^{\delta} \sum_{j=1}^{d} \pi_{\delta, t}^{j, k} \sum_{i=1}^{d} b^{j, i}\left(t, \boldsymbol{Y}_{t}\right)\left(\tilde{\theta}^{i}\left(t, \tilde{\boldsymbol{Z}}_{t}\right) d t+d \tilde{W}_{t}^{i}\right)
$$

the growth rate

$$
\bar{g}_{t}^{\delta, k}=\sum_{i=1}^{d}\left(\sum_{j=1}^{d} \pi_{\delta, t}^{j, k} b^{j, i}\left(t, \boldsymbol{Y}_{t}\right) \tilde{\theta}^{i}\left(t, \tilde{\boldsymbol{Z}}_{t}\right)-\frac{1}{2}\left(\sum_{\ell=1}^{d} \pi_{\delta, t}^{\ell, k} b^{\ell, i}\left(t, \boldsymbol{Y}_{t}\right)\right)^{2}\right)
$$

the fractions for the GOP $S_{t}^{\delta_{*}, k}$

$$
\pi_{\delta_{*}, t}^{j, k}=\sum_{i=1}^{d} \tilde{\theta}^{i}\left(t, \tilde{\boldsymbol{Z}}_{t}\right)\left(b^{-1}\right)^{j, i}\left(t, \boldsymbol{Y}_{t}\right)
$$

the SDE for the discounted value $\bar{S}_{t}^{\delta_{*}, k}=\frac{S_{t}^{\delta_{*}, k}}{S_{t}^{0}}$ of the GOP

$$
d \bar{S}_{t}^{\delta_{*}, k}=\bar{S}_{t}^{\delta_{*}, k} \sum_{i=1}^{d} \tilde{\theta}^{i}\left(t, \tilde{\boldsymbol{Z}}_{t}\right)\left(\tilde{\theta}^{i}\left(t, \tilde{\boldsymbol{Z}}_{t}\right) d t+d \tilde{W}_{t}^{i}\right)
$$

and the SDE for a benchmarked portfolio

$$
d\left(\frac{S_{t}^{\delta}}{S_{t}^{\delta_{*}, k}}\right)=\frac{S_{t}^{\delta}}{S_{t}^{\delta_{*}, k}} \sum_{j=1}^{d} \pi_{\delta, t}^{j, k} \sum_{i=1}^{d}\left(b^{j, i}\left(t, \boldsymbol{Y}_{t}\right)-\tilde{\theta}^{i}\left(t, \tilde{\boldsymbol{Z}}_{t}\right)\right) d \tilde{W}_{t}^{i} .
$$

Finally, it follows for the optimal fractions of the GOP under partial information that

$$
\pi_{\delta_{*}, t}^{j, k}=E\left(\pi_{\delta_{*}, t}^{j} \mid \mathcal{A}_{t}^{k}\right)
$$


This provides a rather intuitive relationship between the GOP $S^{\delta_{*}}$ under full information and the GOP $S^{\delta_{*}, k}$ under partial information.

By using equality (38) and Jensen's inequality we obtain the following result:

Corollary 5 The optimal growth rates under full and partial information satisfy the inequality

$$
E\left(\bar{g}_{t}^{\delta_{*}}\right)=\frac{1}{2} \sum_{i=1}^{d} E\left(E\left(\left(\theta^{i}\left(t, \boldsymbol{X}_{t}\right)\right)^{2} \mid \mathcal{A}_{t}^{k}\right)\right) \geq \frac{1}{2} \sum_{i=1}^{d} E\left(\left(\tilde{\theta}^{i}\left(t, \tilde{\boldsymbol{Z}}_{t}\right)\right)^{2}\right)=E\left(\bar{g}_{t}^{\delta_{*}, k}\right)
$$

for all $t \in \Re_{+}$.

This shows that the expected squared total market price of risk under full information is larger than that under partial information. This is an intuitive and rather important observation, which says that more information leads to higher expected growth rates in the corresponding GOP. For the following we assume that $\pi_{\delta_{*}, t}^{0, k} \neq 0$ for all $t \in \Re_{+}$, which excludes the trivial case where the GOP $S^{\delta_{*}, k}$ equals the savings account.

\section{$5 \quad$ Locally Optimal Portfolios}

As denoted in Platen \& Heath (2006), most of the classical portfolio optimization criteria lead to locally optimal portfolios. The corresponding theory has so far been developed for the case of full information. Here we want to investigate how locally optimal strategies vary in the case of partial information. As described in Platen (2005), most of the classical setups can be synthesized in the basic problem of maximizing the discounted drift under a fixed level for the value of the aggregate diffusion coefficient.

To deal now with this issue in the case of partial information corresponding to $\underline{\mathcal{A}}^{k}$, we start from the dynamics (32) that we rewrite equivalently in terms of the $\underline{\mathcal{A}}^{k}$-adapted strategy $\boldsymbol{\delta}$, namely

$$
d \bar{S}_{t}^{\delta}=\sum_{j=1}^{d} \delta_{t}^{j} \bar{S}_{t}^{j}\left(\tilde{B}^{j}\left(t, \tilde{\boldsymbol{Z}}_{t}\right)+\sum_{i=1}^{d} b^{j, i}\left(t, \boldsymbol{Y}_{t}\right) d \tilde{W}_{t}^{i}\right)
$$

Notice that both $\delta_{t}^{j}$ and $\bar{S}_{t}^{j}$ are adapted to $\underline{\mathcal{A}}^{k}$ and $\tilde{W}_{t}^{i}$ forms an $\left(\underline{\mathcal{A}}^{k}, P\right)$-Wiener process.

Following Platen (2005), the aggregate diffusion coefficient is

$$
\gamma_{t}^{\delta}=\sqrt{\sum_{i=1}^{d}\left(\sum_{j=1}^{d} \delta_{t}^{j} \bar{S}_{t}^{j} b^{j, i}\left(t, \boldsymbol{Y}_{t}\right)\right)^{2}}
$$


while the discounted drift is simply

$$
\alpha_{t}^{\delta}=\sum_{j=1}^{d} \delta_{t}^{j} \bar{S}_{t}^{j} \tilde{B}^{j}\left(t, \tilde{\boldsymbol{Z}}_{t}\right)
$$

The local portfolio optimization now consists in solving, for each time $t$ and a given value $\bar{\gamma}_{t}$ of the aggregate diffusion coefficient, the

\section{Problem A:}

$$
\left\{\begin{array}{l}
\max _{\delta} \alpha_{t}^{\delta} \\
\left(\gamma_{t}^{\delta}\right)^{2}=\bar{\gamma}_{t}^{2}>0
\end{array}\right.
$$

Typically, this problem can be solved by using the method of Lagrange multipliers, namely

$$
\max _{\boldsymbol{\delta}_{t}, \lambda_{t}} E\left(\sum_{j=1}^{d} \delta_{t}^{j} \bar{S}_{t}^{j} \tilde{B}^{j}\left(t, \tilde{\boldsymbol{Z}}_{t}\right)-\lambda_{t}\left(\sum_{j=1}^{d} \sum_{\ell=1}^{d} \delta_{t}^{j} \delta_{t}^{\ell} \bar{S}_{t}^{j} \bar{S}_{t}^{\ell} A^{j, \ell}\left(t, \boldsymbol{Y}_{t}\right)-\bar{\gamma}_{t}^{2}\right)\right) .
$$

For each $\lambda_{t}$ the expression in (50) is a concave function of the vector $\boldsymbol{\delta}_{t}=$ $\left(\delta_{t}^{0}, \ldots, \delta_{t}^{d}\right)^{\top}$ so that the locally optimal strategy is obtained by solving a system of $d$ linear equations in the $d$ unknowns $\delta_{t}^{j}$ under the condition that

$$
\sum_{j, \ell=1}^{d} \delta_{t}^{j} \delta_{t}^{\ell} \bar{S}_{t}^{j} \bar{S}_{t}^{\ell} A^{j, \ell}\left(t, \boldsymbol{Y}_{t}\right)=\bar{\gamma}_{t}^{2}
$$

Let $\tilde{\boldsymbol{\theta}}\left(t, \tilde{\boldsymbol{Z}}_{t}\right)=\left(\tilde{\theta}^{1}\left(t, \tilde{\boldsymbol{Z}}_{t}\right), \ldots, \tilde{\theta}^{d}\left(t, \tilde{\boldsymbol{Z}}_{t}\right)\right)^{\top}$ denote the vector of filtered market prices of risk, see (38). This allows us to formulate the following portfolio selection theorem.

Theorem 6 Under partial information corresponding to $\underline{\mathcal{A}}^{k}$, the discounted locally optimal portfolio $\bar{S}^{\tilde{\delta}, k}$ that solves the above Problem A satisfies the SDE

$$
d \bar{S}_{t}^{\tilde{\delta}, k}=\bar{S}_{t}^{\tilde{\delta}, k}\left(J_{t}^{\tilde{\delta}}\right)^{-1} \sum_{i=1}^{d} \tilde{\theta}^{i}\left(t, \tilde{\boldsymbol{Z}}_{t}\right)\left(\tilde{\theta}^{i}\left(t, \tilde{\boldsymbol{Z}}_{t}\right) d t+d \tilde{W}_{t}^{i}\right)
$$

with fractions

$$
\pi_{\tilde{\delta}, t}^{j, k}=\frac{\pi_{\delta_{*}, t}^{j, k}}{J_{t}^{\tilde{\delta}}}
$$

$j \in\{1,2, \ldots, d\}$, and a corresponding $\underline{\mathcal{A}}^{k}$-adapted risk aversion coefficient

$$
J_{t}^{\tilde{\delta}}=\frac{\left|\tilde{\boldsymbol{\theta}}\left(t, \tilde{\boldsymbol{Z}}_{t}\right)\right|}{\bar{\gamma}_{t}}>0,
$$

$t \in \Re_{+}$. 
The proof of this theorem is similar to that for the case of full information, as given in Platen \& Heath (2006), and is therefore omitted.

Remark 7 The Problem $A$ becomes analogous to that for the case of full information provided that $\boldsymbol{\theta}\left(t, \boldsymbol{X}_{t}\right)$ is replaced by $\tilde{\boldsymbol{\theta}}\left(t, \tilde{\boldsymbol{Z}}_{t}\right)=E\left(\boldsymbol{\theta}\left(t, \boldsymbol{X}_{t}\right) \mid \mathcal{A}_{t}^{k}\right)$. In particular, a risky locally optimal portfolio invests always a fraction in the GOP $S^{\delta_{*}, k}$ and holds the remainder in the savings account.

Once this basic locally optimal portfolio problem has been solved, its solution can be used to study classical questions in portfolio selection, now under partial information. For risky locally optimal portfolios the Sharpe ratio at time $t$ turns out to equal the total market price of risk $\left|\tilde{\boldsymbol{\theta}}\left(t, \tilde{\boldsymbol{Z}}_{t}\right)\right|$, which follows as in Platen (2005). Similar as in Platen \& Heath (2006), under partial observation the capital market line has its slope at time $t$ given by the Sharpe ratio. Finally, the Markowitz efficient frontier is formed, as in Platen (2005), by the above family of locally optimal portfolios obtained under partial information. According to Theorem 6 the given value $\bar{\gamma}_{t}$ of the diffusion coefficient of the locally optimal portfolio determines at time $t$ the fraction invested in the GOP. The inverse of this fraction can be interpreted as risk aversion coefficient, see Pratt (1964) and Arrow (1965).

These results confirm that also under partial information the classical statements of portfolio theory can be derived.

\section{Expected Utility Maximization under Partial Information}

As described in Platen (2005), it can be shown that expected utility maximization of discounted terminal wealth can be brought into a relationship with the GOP. This results in the corresponding optimal portfolio being also locally optimal. To determine, under the information given by $\underline{\mathcal{A}}^{k}$, the expected utility maximizing strategy $\tilde{\delta}$ we proceed as in the, so-called, martingale approach to portfolio optimization, see, e.g. Karatzas \& Shreve (1998). Here one first determines the terminal portfolio value that maximizes the expected utility and then calculates the strategy that replicates this value. To this effect the notion of a fair portfolio plays a significant role. This is a portfolio that, when expressed in units of the GOP, forms a martingale. It can be shown that, although there may be various nonnegative portfolios that replicate a given future payoff, the minimal portfolio that does so is the fair portfolio. This fact allows for a mathematical characterization in that a generic nonnegative replicating portfolio with strategy $\delta$, when benchmarked by the GOP, is a supermartingale. The fair portfolio, when benchmarked by the GOP is, however, a martingale and, therefore, requires the smallest initial wealth to reach a given target. 
Definition 8 Under partial information, as expressed by the filtration $\underline{\mathcal{A}}^{k}$, a price process $S=\left\{S_{t}, t \in \Re_{+}\right\}$is called fair when its benchmarked value $\hat{S}_{t}=\frac{S_{t}}{S_{t}^{\delta_{*}, k}}$ forms an $\left(\underline{\mathcal{A}}^{k}, P\right)$-martingale.

Given a utility function $U(\cdot)$ which is strictly increasing, strictly concave, satisfies $U^{\prime}(0)=\infty$ and $U^{\prime}(\infty)=0$ in addition to being twice differentiable and given a situation of partial information as expressed by the filtration $\underline{\mathcal{A}}^{k}$, our problem here is the following

Problem B: Maximize $E\left(U\left(\bar{S}_{T}^{\delta}\right) \mid \mathcal{A}_{0}^{k}\right)$ over all $\underline{\mathcal{A}}^{k}$-adapted strategies $\delta$, where $\bar{S}_{T}^{\delta}$ denotes the discounted terminal value of a fair portfolio corresponding to an $\underline{\mathcal{A}}^{k}$-adapted strategy $\delta$ with budget constraint $S_{0}^{\delta}=S_{0}>0$.

This leads to the following result:

Proposition 9 Assuming that the benchmarked savings account $\hat{S}_{t}^{0, k}=\frac{S_{t}^{0}}{S_{t}^{\delta, k}}$ under partial information is a scalar Markov process, the $\underline{\mathcal{A}}^{k}$-adapted discounted portfolio $\bar{S}_{t}^{\tilde{\delta}, k}$ that solves Problem $B$ is given by the $S D E$

$$
\frac{d \bar{S}_{t}^{\tilde{\delta}, k}}{\bar{S}_{t}^{\tilde{\delta}, k}}=\left(J_{t}^{\tilde{\delta}}\right)^{-1} \frac{d \bar{S}_{t}^{\delta_{*}, k}}{\bar{S}_{t}^{\delta_{*}, k}}
$$

for $t \in \Re_{+}$with risk aversion coefficient

$$
J_{t}^{\tilde{\delta}}=\left(1-\frac{\hat{S}_{t}^{0, k}}{\hat{u}\left(t, \hat{S}_{t}^{0, k}\right)} \frac{\partial \hat{u}\left(t, \hat{S}_{t}^{0, k}\right)}{\partial \hat{S}^{0, k}}\right)^{-1}
$$

and benchmarked portfolio value

$$
\hat{u}_{\lambda}\left(t, \hat{S}_{t}^{0, k}\right)=\frac{\bar{S}_{t}^{\tilde{\delta}}}{\bar{S}_{t}^{\delta_{*}, k}}=E\left(\left(U^{\prime}\right)^{-1}\left(\frac{\lambda}{\bar{S}_{T}^{\delta_{*}, k}}\right) \frac{1}{\bar{S}_{T}^{\delta_{*}, k}} \mid \mathcal{A}_{t}^{k}\right)
$$

where $\lambda$ is such that $S_{0}^{\tilde{\delta}}=S_{0}$.

Proof of Proposition 9: $\quad$ Notice first that the discounted terminal value $\bar{S}_{T}^{\tilde{\delta}}$ of a fair portfolio corresponding to an $\underline{\mathcal{A}}^{k}$-adapted strategy $\tilde{\delta}$ that maximizes $E\left(U\left(\bar{S}_{T}^{\delta}\right) \mid \mathcal{A}_{t}^{k}\right)$ among all $\underline{\mathcal{A}}^{k}$-adapted strategies can be obtained, similarly as in Platen \& Heath (2006), as

$$
\bar{S}_{T}^{\tilde{\delta}}=\left(U^{\prime}\right)^{-1}\left(\frac{\lambda}{\bar{S}_{T}^{\delta_{*}, k}}\right)
$$

where $\left(U^{\prime}\right)^{-1}$ is the inverse of the first derivative of $U$ and $T>0$, the maturity. Here the parameter $\lambda$ is chosen so as to satisfy the budget constraint given by 
the initial wealth $S_{0}^{\tilde{\delta}}=S_{0}$. Recall that by $\bar{S}_{t}^{\delta_{*}, k}$ we denote the discounted, with respect to $S_{t}^{0}$, value of the GOP corresponding to the partial information $\underline{\mathcal{A}}^{k}$ and set $\mathcal{A}_{0}^{k}=\mathcal{A}_{0}$. Since $S^{\tilde{\delta}}$ shall be fair, the condition on the parameter $\lambda$ can, therefore, be expressed by requiring that

$$
E\left(\left(U^{\prime}\right)^{-1}\left(\frac{\lambda}{\bar{S}_{T}^{\delta_{*}, k}}\right) \frac{1}{\bar{S}_{T}^{\delta_{*}, k}} \mid \mathcal{A}_{0}^{k}\right)=\frac{S_{0}^{\tilde{\delta}}}{S_{0}^{\delta_{*}, k}}=\frac{S_{0}}{S_{0}^{\delta_{*}, k}} .
$$

This explains the $\left(\underline{\mathcal{A}}^{k}, P\right)$-martingale $\hat{u}_{\lambda}\left(t, \hat{S}_{t}^{0, k}\right)$ in $(57)$, which is a function of $t$ and the scalar Markov process $\hat{S}_{t}^{0, k}$. The budget constraint then becomes

$$
\hat{u}_{\lambda}\left(0, \hat{S}_{0}^{0, k}\right)=\frac{S_{0}}{S_{0}^{\delta_{*}, k}}
$$

The requested strategy $\tilde{\delta}$ is now the strategy that hedges the payoff under the conditional expectation on the right hand side of (57). To this effect we use the dynamics of the Markovian benchmarked savings account to form a benchmarked self-financing portfolio and then identify the diffusion coefficient which yields the risk aversion coefficient in (56). The remainder of the proof is similar to that in Platen \& Heath (2006) for the case of full information.

We remark that when dropping the assumption on the Markovianity of $\hat{S}^{0, k}$ one still obtains the benchmarked portfolio value as in (57). However, the strategy is more complex.

\section{Utility Indifference Pricing under Partial Information}

The combination of portfolio optimization and hedging under partial information, which we presented in the previous section and for which we required a portfolio to be fair, allows us to deal also with the pricing of nonreplicable contingent claims under partial information. For this purpose we will apply the concept of utility indifference pricing, see Davis (1997). The utility indifference price is the price at which the investor is indifferent between adding a small part of a contract to his or her portfolio or continuing the investment strategy that maximizes his or her expected utility. This approach acknowledges the fact that the pricing of a contract is, in principle, an investment decision.

To be precise, we consider now a contingent claim with discounted payoff $\bar{H}$ at time $T \in \Re_{+}$. Let us denote by $V_{t}^{k}$ the hypothetical purchasing price for the claim at time $t \in[0, T]$ under the partial information corresponding to $\underline{\mathcal{A}}^{k}$. We assume that the investor buys a vanishing fraction $\varepsilon>0$ of the contract at time $t$ for the small total amount $\varepsilon V_{t}^{k}$. Beyond this purchase, the investor continues 
to invest the major part of her or his wealth according to the expected utility maximizing strategy $\tilde{\delta}$ described in the previous section.

Similarly as in Platen \& Heath (2006) we introduce for $\varepsilon \geq 0$ the following expected utility

$$
v_{\varepsilon, V_{t}^{k}}^{\tilde{\delta}, k}=E\left(U\left(\left(S_{t}-\varepsilon V_{t}^{k}\right) \frac{\bar{S}_{T}^{\tilde{\delta}}}{S_{t}}+\varepsilon \bar{H}\right) \mid \mathcal{A}_{t}^{k}\right) .
$$

Here $S_{t}$ denotes the total wealth of the investor at time $t$, which provides the budget constraint. The amount $S_{t}-\varepsilon V_{t}^{k}$ is then at time $t$ invested according to the expected utility maximizing strategy $\tilde{\delta}$. Therefore, at the maturity date $T$ the discounted payoff $\varepsilon \bar{H}$ is obtained additionally to the discounted portfolio value $\left(S_{t}-\varepsilon V_{t}^{k}\right) \frac{\bar{S}_{T}^{\delta}}{S_{t}}$ resulting from the investment using the strategy $\tilde{\delta}$.

Definition 10 The value $V_{t}^{k}$ is called utility indifference price for $\bar{H}$ at time $t$ if

$$
\lim _{\varepsilon \rightarrow 0} \frac{v_{\varepsilon, V_{t}^{k}}^{\tilde{\delta}, k}-v_{0, V_{t}^{k}}^{\tilde{\delta}, k}}{\varepsilon}=0
$$

almost surely.

According to this definition, the expected utility of the investor does not change much if the price at time $t$ for the payoff $\bar{H}$ is chosen to be $V_{t}^{k}$. In this sense the utility indifference price is consistent with the overall expected utility maximizing investment strategy.

It is now of interest to clarify what formula results for the utility indifference price. In principle, this depends on the underlying model, the utility function, the information level and also the payoff. However, by letting $\varepsilon$ tend to zero we will see that a simple general pricing rule, the real world pricing formula under partial information, emerges rather generally for nonreplicable payoffs $H=\bar{H} S_{T}^{0}$.

Proposition 11 Under appropriate technical assumptions on the payoff, utility function and the model, the utility indifference price $V_{t}^{k}$ at time $t$ for a claim $H=\bar{H} S_{T}^{0}$ is given by the real world pricing formula under partial information

$$
V_{t}^{k}=S_{t}^{\delta_{*}, k} E\left(\frac{H}{S_{T}^{\delta_{*}, k}} \mid \mathcal{A}_{t}^{k}\right)
$$

Recall that the payoff $H$ in (62) may be nonreplicable. The formula (62) is here called the real world pricing formula under partial information since it generalizes the real world pricing formula under full information in the sense of Platen 
\& Heath (2006). Only the real world probability $P$ is used to calculate the expectation (62). This is a satisfying result since it states the independence of the pricing concept from the subjective utility functions of investors.

From (62) follows then the martingale relation

$$
\frac{V_{t}}{S_{t}^{\delta_{*}, k}}=E\left(\frac{H}{S_{T}^{\delta_{*}, k}} \mid \mathcal{A}_{t}^{k}\right)
$$

for $t \in[0, T]$. Therefore, the benchmarked price $\frac{V_{t}}{S_{t}^{\delta_{*}, k}}$ is obtained as an expectation under the real world probability measure $P$ of the benchmarked payoff $\frac{H}{S_{T}^{\delta_{*}, k}}$ conditional on the partial information $\mathcal{A}_{t}^{k}$ available at time $t \in[0, T]$. To be precise, $\frac{V_{t}}{S_{t}^{\delta_{*}, k}}$ forms an $\left(\underline{\mathcal{A}}^{k}, P\right)$-martingale and is, thus, a fair price process with respect to the partial information structure given by the filtration $\mathcal{A}^{k}$. The numeraire is here the GOP under partial information $S^{\delta_{*}, k}$. Note that the existence of an equivalent risk neutral probability measure is not required under the above benchmark approach. When trying to deal with partial information in a classical risk neutral setting one soon realizes that this becomes technically extremely demanding and finally remains less general than what we obtain in a straightforward manner under the benchmark approach.

Proof of Proposition 11 The proof of the real world pricing formula (62) requires to satisfy a number of technical conditions that are straightforward but best formulated for particular models, payoffs and utilities. Therefore, we outline in the following only the essential steps that lead to formula (62). For simplicity of notation we shall drop the superscripts $k$ in $V_{t}^{k}$ and $v_{\varepsilon, V_{t}^{k}}^{\tilde{\delta}, k}$. First let us expand the utility function under the expectation in (60) by the Taylor formula. Neglecting higher order terms in $\varepsilon$ yields:

$$
\begin{aligned}
v_{\varepsilon, V_{t}}^{\tilde{\delta}} & \approx E\left(U\left(\bar{S}_{T}^{\tilde{\delta}}\right)+U^{\prime}\left(\bar{S}_{T}^{\tilde{\delta}}\right) \varepsilon\left(\bar{H}-V_{t} \frac{\bar{S}_{T}^{\tilde{\delta}}}{S_{t}}\right) \mid \mathcal{A}_{t}^{k}\right) \\
& =v_{0, V_{t}}^{\tilde{\delta}}+\varepsilon E\left(U^{\prime}\left(\bar{S}_{T}^{\tilde{\delta}}\right)\left(\bar{H}-V_{t} \frac{\bar{S}_{T}^{\tilde{\delta}}}{S_{t}}\right) \mid \mathcal{A}_{t}^{k}\right) .
\end{aligned}
$$

Using the asymptotic condition (61) together with (64), allows us to identify the utility indifference price via the relation

$$
0=\lim _{\varepsilon \rightarrow 0} \frac{v_{\varepsilon, V_{t}}^{\tilde{\delta}}-v_{0, V_{t}}^{\tilde{\delta}}}{\varepsilon}=E\left(U^{\prime}\left(\bar{S}_{T}^{\tilde{\delta}}\right)\left(\bar{H}-V_{t} \frac{\bar{S}_{T}^{\tilde{\delta}}}{S_{t}}\right) \mid \mathcal{A}_{t}^{k}\right) .
$$

By solving this equation for $V_{t}$ we obtain

$$
V_{t}=\frac{E\left(U^{\prime}\left(\bar{S}_{T}^{\tilde{\delta}}\right) \bar{H} \mid \mathcal{A}_{t}^{k}\right)}{E\left(U^{\prime}\left(\bar{S}_{T}^{\tilde{\delta}}\right) \frac{\bar{S}_{T}^{\tilde{\delta}}}{S_{t}} \mid \mathcal{A}_{t}^{k}\right)}
$$


Exploiting the fact that at maturity $T$ the discounted expected utility maximizing portfolio value $\bar{S}_{T}^{\tilde{\delta}}$ equals by $(58)$ the random variable $\left(U^{\prime}\right)^{-1}\left(\frac{\lambda}{\bar{S}_{T}^{\delta_{*}, k}}\right)$, in formula (65) the factors $U^{\prime}(\cdot)$ and $\left(U^{\prime}\right)^{-1}(\cdot)$ offset each other in the numerator as well as in the denominator. Consequently, the formula turns out to be independent of the subjective utility function of the investor. More precisely, we obtain from (65)

$$
V_{t}=\frac{E\left(\frac{\lambda}{\bar{S}_{T}^{\delta_{*}, k}} \bar{H} \mid \mathcal{A}_{t}^{k}\right)}{E\left(\frac{\lambda}{\bar{S}_{T}^{\delta_{*}, k}} \frac{\bar{S}_{T}^{\tilde{\delta}}}{S_{t}} \mid \mathcal{A}_{t}^{k}\right)}=\frac{S_{t}^{\delta_{*}, k} E\left(\frac{H}{S_{T}^{\delta_{*}, k}} \mid \mathcal{A}_{t}^{k}\right)}{\frac{S_{t}^{\delta_{*}, k}}{S_{t}} E\left(\frac{S_{T_{T}}^{\tilde{\delta}}}{S_{T}^{\delta_{*}, k}} \mid \mathcal{A}_{t}^{k}\right)} .
$$

Since $S^{\tilde{\delta}}$ has to be a fair portfolio with initial value $S_{t}$ at time $t$, the denominator on the right hand side of the last equation yields the value one. Therefore, one obtains for the given nonreplicable payoff under partial observation the utility indifference pricing formula (62). Of course, appropriate technical conditions need to be satisfied for the considered particular case that allow us to perform the above steps. For instance, for the Black-Scholes model, see Black \& Scholes (1973), and the Minimal Market Model, see Platen (2001), as models for the discounted GOP in combination with some power utility function, such conditions are satisfied.

\section{Conclusions}

The paper has demonstrated that portfolio optimization can be efficiently performed under the benchmark approach when only partial information is available. The particular study of locally optimal portfolios allowed us to relate the results to the classical theory of portfolio selection. Furthermore, in this context the optimal strategy for maximizing expected utility from discounted terminal wealth under partial information requires investing only in the growth optimal portfolio and in the savings account. In addition it turns out that nonreplicable payoffs can be consistently evaluated via expected utility indifference pricing under partial information, yielding a real world pricing formula under partial information. Here the GOP is the numeraire and the pricing measure is the real world probability measure. The existence of an equivalent risk neutral probability measure is not required.

\section{Acknowledgement}

The second author would like to thank the Quantitative Finance Research Centre at UTS for its hospitality. Furthermore, support from the ARC grant DP 0559879 is acknowledged. 


\section{References}

Arrow, K. J. (1965). Aspects of the Theory of Risk-Bearing. Helsinki: Yrjö Hahnsson Foundation.

Babbs, S. H. \& K. B. Nowman (1999). Kalman filtering of generalized Vasicek term structure models. J. Financial and Quantitative Analysis 34, 115-130.

Bäuerle, N. \& U. Rieder (2007). Portfolio optimization with jumps and unobservable intensity process. Math. Finance. to appear.

Bhar, R., C. Chiarella, \& W. Runggaldier (2004). Inferring the forward looking equity risk premium from derivative prices. Studies in Nonlinear Dynamics \& Econometrics 8(1). Article 3, http://www.bepress.com/snde/vol8/iss1/art3.

Black, F. \& M. Scholes (1973). The pricing of options and corporate liabilities. J. Political Economy 81, 637-654.

Cvitanić, J., B. Rozovskii, \& I. Zaliapin (2006). Numerical estimation of volatility values from discretely observed diffusion data. J. Comput. Finance 9(4), ??-??

Davis, M. H. A. (1997). Option pricing in incomplete markets. In M. A. H. Dempster and S. R. Pliska (Eds.), Mathematics of Derivative Securities, pp. 227-254. Cambridge University Press.

Elliott, R. J., P. Fischer, \& E. Platen (1999). Hidden Markov filtering for a mean reverting interest rate model. In 38th IEEE CDC meeting in Phoenix, pp. 2782-2787. IEEE Control Systems Society.

Elliott, R. J., C. H. Lahaie, \& D. B. Madan (1997). Filtering derivative security valuations from market prices. In M. A. H. Dempster and S. R. Pliska (Eds.), Mathematics of Derivative Securities, pp. 141-162. Cambridge University Press.

Frey, R. \& W. J. Runggaldier (1999). Risk minimizing hedging strategies under restricted information: The case of stochastic volatility models observable only at random discrete times. Math. Methods Oper. Res. 50, 339-350.

Frey, R. \& W. J. Runggaldier (2001). A nonlinear filtering approach to volatility estimation with a view towards high frequency data. Int. J. Theor. Appl. Finance 4(2), 199-210.

Gombani, A., S. R. Jaschke, \& W. J. Runggaldier (2005). A filtered no arbitrage model for term structures from noisy data. Stochastic Process. Appl. 115, 381-400.

Haussmann, U. G. \& J. Sass (2004). Optimizing the terminal wealth under partial information: The drift process as a continuous time Markov chain. Finance Stoch. 8, 553-577.

Karatzas, I. \& S. E. Shreve (1998). Methods of Mathematical Finance, Volume 39 of Appl. Math. Springer. 
Karatzas, I. \& X. Zhao (2001). Bayesian adaptive portfolio optimization. In E. Jouini, J. Cvitanić, and M. Musiela (Eds.), Handbook Math. Finance: Option pricing, interest rates and risk management, pp. 632-669. Cambridge University Press.

Kirch, M. \& W. J. Runggaldier (2004). Efficient hedging when asset prices follow a geometric Poisson process with unknown intensities. SIAM J. Control Optim. 43, 1174-1195.

Lakner, P. (1995). Utility maximization with partial information. Stochastic Process. Appl. 56(2), 247-273.

Landen, C. (2000). Bond pricing in a hidden Markov model of the short rate. Finance Stoch. 4, 371-389.

Nagai, H. \& S. Peng (2002). Risk-sensitive dynamic portfolio optimization with partial information on infinite time horizon. Ann. Appl. Probab. 12, 173195.

Pham, H. \& M. C. Quenez (2001). Optimal portfolio in partially observed stochastic volatility models. Ann. Appl. Probab. 11, 210-238.

Platen, E. (2001). A minimal financial market model. In Trends in Mathematics, pp. 293-301. Birkhäuser.

Platen, E. (2005). Diversified portfolios with jumps in a benchmark framework. Asia-Pacific Financial Markets 11(1), 1-22.

Platen, E. \& D. Heath (2006). A Benchmark Approach to Quantitative Finance. Springer Finance. Springer.

Platen, E. \& W. J. Runggaldier (2005). A benchmark approach to filtering in finance. Asia-Pacific Financial Markets 11(1), 79-105.

Pratt, J. W. (1964). Risk aversion in the small and in the large. Econometrica 32, 122-136.

Runggaldier, W. J. (2004). Estimation via stochastic filtering in financial market models. Contemp. Math. 351, 309-318. 